\title{
Absolute Radial Velocities by Cross-Correlation with Synthetic Spectra
}

\author{
J. Skuljan, J. B. Hearnshaw and P. L. Cottrell \\ Department of Physics and Astronomy, University of Canterbury, \\ Private Bag 4800, Christchurch, New Zealand
}

\begin{abstract}
Precise absolute radial velocities have been measured for several hundred late-type stars at Mt John University Observatory with the 1-m telescope, fiber-fed échelle spectrograph, and Thomson CCD. Six échelle orders in the green (5000-5600 $\AA$ ) are used. Many delicate steps have been undertaken in order to maintain exactly the same conditions, both in recording and reducing the spectra, over a period of 18 months. A cross-correlation technique with theoretical spectra computed by $R$. L. Kurucz has been chosen to determine the absolute radial velocities. Blue sky spectra have been used to monitor systematic zero-point fluctuations from one observing run to another. An additional correlation between the measured velocities and average number of $A / D$ units in the continuum has been discovered and used for fine adjustments, significantly improving the results. A random uncertainty of $10-20 \mathrm{~m} \mathrm{~s}^{-1}$ has been achieved for stellar spectra having intrinsically constant radial velocities.
\end{abstract}

\section{Introduction}

Absolute radial velocity measurements are part of a larger project (Skuljan et al. 1997) started at the University of Canterbury in order to test the reality of moving groups proposed by Olin Eggen. There are about eight hundred stars on the program list. The idea is to get precise radial velocities for these stars, and then combine them with the Hipparcos parallaxes and proper motions in order to compute the space velocities and test the moving group hypothesis.

The observations described here have been done at Mt John University Observatory (MJUO), New Zealand, using the 1-m telescope, fiber-fed échelle spectrograph and a Thomson $576 \times 384$ CCD. The spectral region covered is from $5000 \AA$ to $5600 \AA$ in orders $41-46$. Late type stars (F5-M5) were observed from 1995 October till 1997 March (18 months) without any changes in the instrumental settings. The spectra have a typical $S / N$ ratio of about 100 , at a resolving power $(\lambda / \Delta \lambda)$ of 38000 and dispersion of $24 \mathrm{pix} / \AA$, or $2400 \mathrm{~m} \mathrm{~s}^{-1} / \mathrm{pix}$.

\section{Reduction procedure}

A standard reduction procedure has been performed on two-dimensional raw CCD images using the ESO MIDAS package. A Th-Ar comparison lamp is used 
for wavelength calibration and it is exposed immediately before and after the stellar exposure. A white lamp is used for flat-fielding.

After the cosmic rays have been removed and the background scattered light has been subtracted, the one-dimensional spectra are extracted by following the échelle orders on a 2-D image. Flat-fielding is then performed on 1-D spectra. Since 1-D spectra are formed by averaging several pixels in a direction perpendicular to the dispersion, this means that real pixel-to-pixel variations are not eliminated properly with 1-D flat-fielding. However, this technique is preferable when the optical fiber is used, since the white lamp 2-D spectrum has exactly the same profile as a stellar one, with not enough signal in the wings. Any 2-D flat-fielding would degrade the $\mathrm{S} / \mathrm{N}$ ratio significantly. On the other hand, even if $1-\mathrm{D}$ flat-fielding does not deal with real pixel-to-pixel variations in a proper way, it does remove all large-scale defects, such as the dust patches on the CCD window.

Wavelength calibration is performed using the eight-digit thorium wavelengths by Palmer \& Engleman (1983). The actual line selection has been done with special care, in order to minimize any instabilities in the dispersion solution. Many thorium images have been used to examine the stability in pixel position for each spectral line. By 'stability' here we mean how closely the pixel position of a thorium line will repeat from one image to another if a gaussian profile is fitted to the line always in the same way (using the same number of pixels to the left and right of the central position). There is a correlation between the line position scatter and line intensity, as shown in Figure 1A. Each point represents a spectral line. Pixel positions for all lines have been determined on many images and reduced for any systematic shifts from one image to another. The remaining positions fluctuate as a result of random errors only. We define the position scatter as the standard deviation of all positions found for one particular line. This plot allowed us to eliminate those lines that show unusual scatters out of the general trend. Only the final selection of lines is shown in Figure 1A. Note that for most of the lines, the scatter is about a few hundredths of a pixel, which is less than $50 \mathrm{~m} \mathrm{~s}^{-1}$ or so.
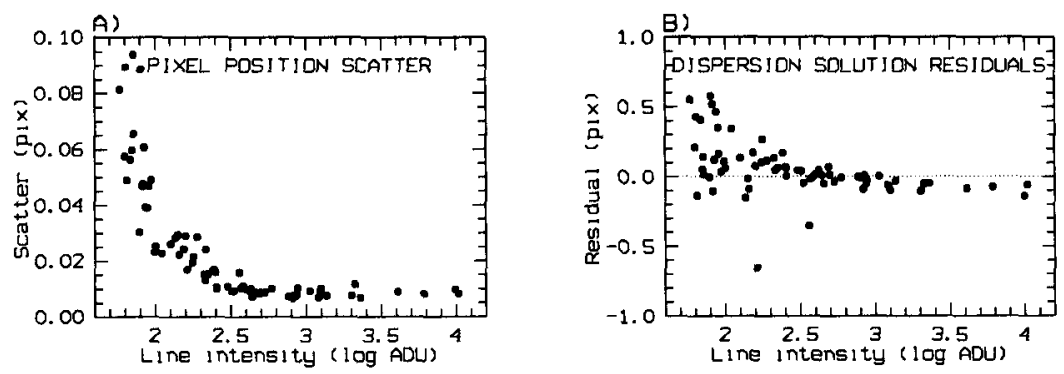

Figure 1. A) Scatter in pixel position versus line intensity. B) Residuals for a $2-\mathrm{D}$ dispersion solution.

A 2-D polynomial is fitted to about 50 lines in all six échelle orders simultaneously, taking the pixel positions as functions of wavelength and order number. Residuals are computed for each line as differences between the measured and 
fitted pixel positions. A typical plot of residuals versus line intensity is presented in Figure 1B. The r.m.s. error is about 0.07 pix. If we divide this value by $\sqrt{N}$, where $N=50$ (total number of lines) and transform the result into velocity units, it would seem that a precision of no better than $25 \mathrm{~m} \mathrm{~s}^{-1}$ can be expected from our wavelength calibration alone. However, this is not the case. We can easily see that the scatter of residuals (Figure $1 \mathrm{~B}$ ) is much larger than the scatter in line positions (Figure 1A), due to blending effects. In fact, if we repeat the wavelength calibration on many thorium images, we will see that each line tends to keep its residual exactly the same (within the random scatter of a few hundredths of a pixel). The wavelength calibration is far more stable than it is suggested by the r.m.s. error of the fit. A typical uncertainty of about $2 \mathrm{~m} \mathrm{~s}^{-1}$ has been found by analyzing many thorium images.

Another characteristic of the residual distribution (Figure 1B) is an unexpected but obvious correlation between the residuals and line intensities. This means that weak lines tend to be shifted to higher pixel positions more than the stronger lines. This peculiarity is a characteristic of our equipment and does not apply in general. It originates in the CCD camera during the readout and is a result of a poor charge transfer efficiency. A certain number of electrons is always left behind, contaminating the following pixels. After a detailed examination we have found that all spectral lines are slightly asymmetric, with longer 'tails' extending towards higher pixel positions. In an absolute amount, the tails contain approximately the same signal that does not depend on line intensities, but in a relative sense, they affect weak lines more than strong ones. The effect becomes opposite when the CCD is rotated by 180 degrees, proving that the problem originates in the camera itself and not in the other parts of the equipment or the reduction procedure.

We are now going to demonstrate that this problem with the CCD camera does not necessarily mean any degradation in final radial velocity precision. First of all, it is important that the same selection of thorium lines is used all the time (the zero-point difference between weak and strong lines can be as high as $300 \mathrm{~m} \mathrm{~s}^{-1}$. The same average number of ADU for all thorium images is also desirable. We have been using the same exposure times, but the average signal has not remained constant, due to some fluctuations affecting the Th-Ar lamp as well as the optical fiber itself.

\section{Synthetic spectra}

After the wavelength calibration and normalization, the 1-D stellar spectrum is cross-correlated with a theoretical (synthetic) spectrum of a similar spectral type. The cross-correlation is done separately for each échelle order and the results are averaged. We use seven different models, computed for this project by R. L. Kurucz (1996). We always choose the model closest to the stellar spectrum in the $\mathrm{H}-\mathrm{R}$ diagram. Both the model and the stellar spectrum are re-normalized so that they have a zero mean and their ends are modified using a cosine bell function (Simkin 1974). 


\section{Radial velocities}

Blue sky spectra have been observed in order to calibrate the stellar velocities. More than 200 sky velocities are presented in Figure 2A. They show a correlation with the average signal in the continuum as well as the thorium intensity used for the wavelength calibration. This is again a result of the charge transfer inefficiency described in Section 2. For a given thorium intensity, there is a smooth (parabolic) dependence on the signal in the continuum. It is possible to fit a 2-D low-order polynomial to these velocities, using the signal in the continuum and thorium intensity as independent variables. Corresponding residuals can then be taken as final reduced velocities of the Sun, randomly fluctuating around $0 \mathrm{~km} \mathrm{~s}^{-1}$, as shown in Figure $2 B$, with an overall uncertainty of $11 \mathrm{~m} \mathrm{~s}^{-1}$ over 18 months. Some additional systematic shifts between different observing runs have also been detected and included in this reduction procedure.
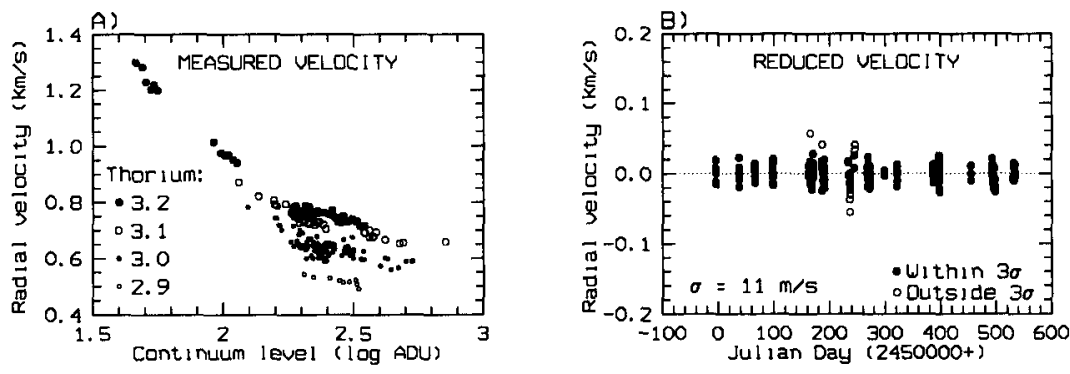

Figure 2. A) Measured radial velocities for the blue sky show an obvious correlation both with the signal in the continuum and thorium intensity. Different symbols are used to represent points with different mean thorium intensities (on a logarithmic scale). B) Reduced velocities for the sky show a random scatter of $11 \mathrm{~m} \mathrm{~s}^{-1}$. Open circles represent the points outside the $3 \sigma$ region. These points have been excluded from the statistics.

We have used the same transformation derived for our sky velocities to reduce all the other stellar spectra. This means that all results have been brought to a system in which the flux spectrum of the Sun has a velocity equal to zero. Some of the IAU standard RV stars are presented in Figure 3. Most of them show some level of variability, in agreement with results already published by other authors. However, there are several stars showing significantly lower scatter (even below $20 \mathrm{~m} \mathrm{~s}^{-1}$ ). These are, for example, the two G5II standards: $\beta$ Lep $\left(\sigma=16 \mathrm{~ms}^{-1}\right)$ and $\beta \operatorname{Crv}\left(\sigma=18 \mathrm{~m} \mathrm{~s}^{-1}\right)$.

Finally, in order to bring our measurements to the standard IAU system, we have computed the residual velocities (measured minus standard) for all standard stars that have been observed. These residuals are presented in Figure 4. We find a standard deviation of $\sigma \approx 400 \mathrm{~m} \mathrm{~s}^{-1}$ with no significant zero-offset. 

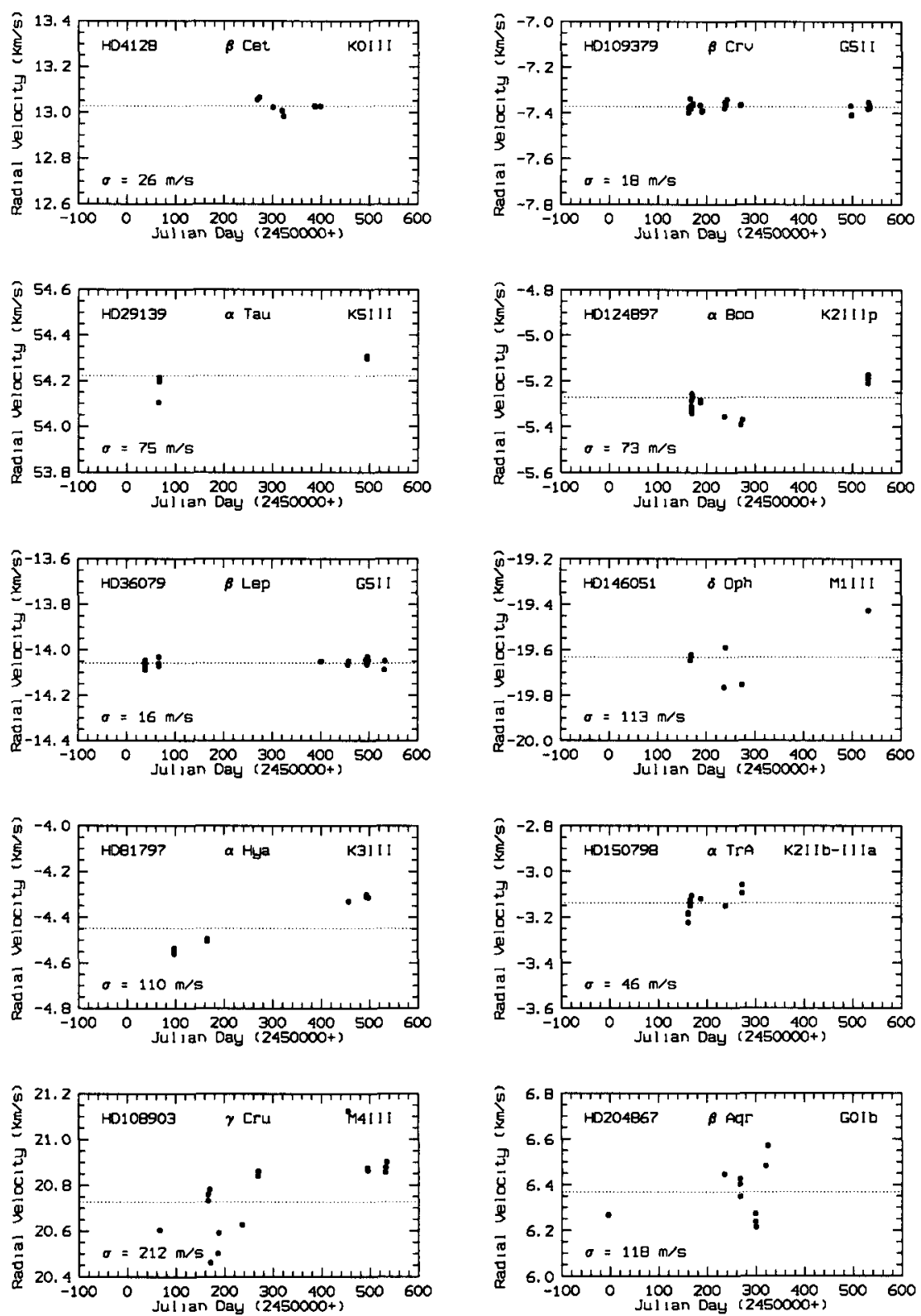

Figure 3. Measured radial velocities for some standard stars. A horizontal dashed line is used to mark the mean value. 

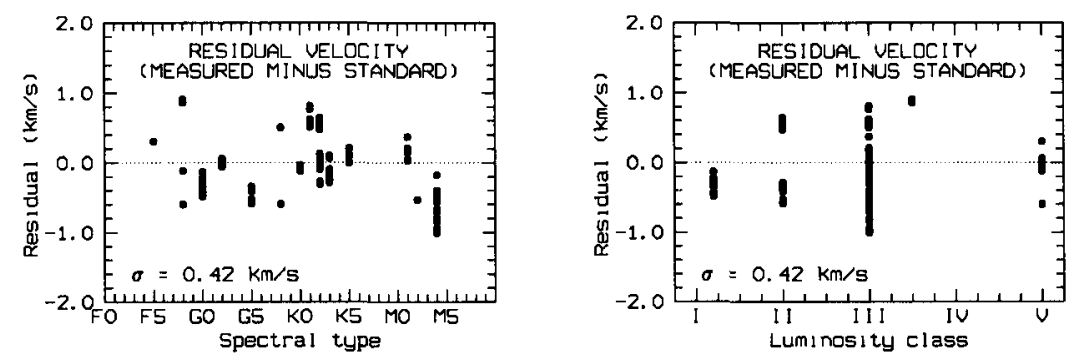

Figure 4. Residual velocities (measured minus standard) versus spectral type and luminosity class.

\section{Discussion}

Batten: The matter of IAU radial-velocity standards was discussed over several years by Commission 30. R. M. Petrie used to quote H. D. Curtis as saying: "There is more joy in heaven over one star that is found to be constant in velocity, than over ninety and nine that are found to be variable!"

Griffin: Your radial-velocity plot for Arcturus demonstrated a small but perhaps not insignificant scatter. In view of a recent publication that Arcturus is a double star, would you care to comment?

Skuljan: We have not got enough measurements to make a firm statement on the nature of the variability, but I am sure that Arcturus is a radial-velocity variable star.

Gullberg: Regarding the title Absolute radial velocities ..., have you been taking into account the net effect of gravitational redshift and convective blueshift in your computations?

Skuljan: No, we have. not.

Irwin: I was surprised that you had some examples of any stable giants $\left(\sigma_{\mathrm{RV}} \sim\right.$ $\left.15 \mathrm{~m} \mathrm{~s}^{-1}\right)$. What fraction of your sample of giants is stable?

Skuljan: Out of 13 giants or supergiants (spectral type from G0 to M4) that I have observed, only two are stable within $15-20 \mathrm{~m} \mathrm{~s}^{-1}$, and they are both classified as G5 II.

Marcy: Could you describe the accuracy of the absolute wavelengths of the absorption lines in the synthetic spectra from R. Kurucz?

Skuljan: I cannot comment on any systematic offset, if it exists, in the wavelengths. However the synthetic lines are all centred on a bin, which is about 0.01 Awide. There may therefore be random errors in the line positions of about this amount. We hope that this problem disappears, on average, when many lines are used.

Popper: How sensitive are the velocities to the match of spectral type and luminosity of the synthetic star spectrum to that of the program star? 
Skuljan: Different synthetic spectra give different radial velocities for the same star, with differences up to about $100 \mathrm{~ms}^{-1}$.

\section{References}

Kurucz, R. L. 1996, private communication

Palmer, B. A., \& Engleman, R. Jr. 1983, Atlas of the Thorium Spectrum, Los Alamos National Laboratory, LA-9615

Simkin, S. M. 1974, A\&A, 31, 129

Skuljan, J., Cottrell, P. L. \& Hearnshaw, J. B. 1997, Proceedings of the ESA Symposium 'Hipparcos - Venice '97', ESA SP-402, p. 525 